\title{
APS-TD Helium Cryogenic Facilities
}

\section{Cryogenic Operations Department}

FERMILAB-POSTER-20-006-TD

\section{Cryomodule Test Facility}

Cryogenic System:

- CMTF cryogenic system is supplied by a state of the art Superfluid Cryogenic Plant to supply $40 \mathrm{~K}, 5 \mathrm{~K}$, and $2 \mathrm{~K}$ helium up to $500 \mathrm{~W}$. $2 \mathrm{~K}$ operations achievable through cold or warm compression systems.

Currently Supports:

- Cryo Module Test Stand 1 (CMTS1) used to test $1.3 \mathrm{GHz}$ \& 3.9 $\mathrm{GHz}$ cryomodules for the LCLS-II project.

- PIP-II Injection Test Stand (PIP-II IT) program to test the front end of the future PIP-II Linac.and production cryomodules
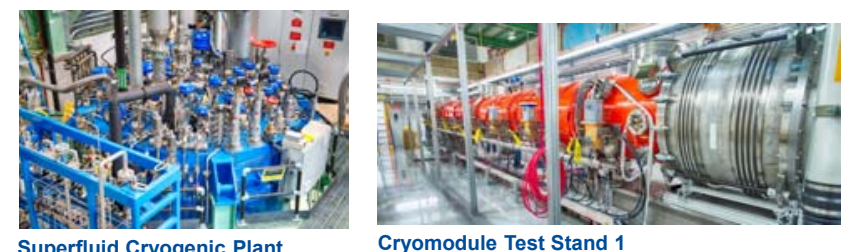

Superfluid Cryogenic Plant

New Muon Lab (NML)

Cryogenic System:

Two Tevatron satellite refrigerators capable of $125 \mathrm{~L} / \mathrm{hr} 4.5 \mathrm{~K}$ liquefaction rate. Kinney Vacuum Pump enables $2 \mathrm{~K}$ operations with maximum $2 \mathrm{~K}$ capacity of $\sim 60 \mathrm{~W}$.

Currently Supports:

NML Cryogenic System cools a cryomodule and two capture cavities to support the Integrable Optics Test Accelerator (IOTA) ring to support accelerator R\&D through $150 \mathrm{MeV}$ electrons from RF linear accelerator

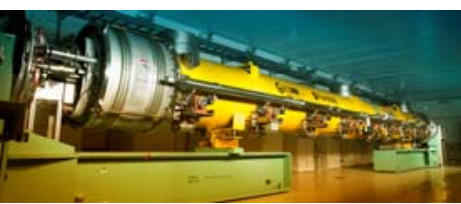

NML Cryomodule

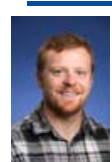

슬

Hurd

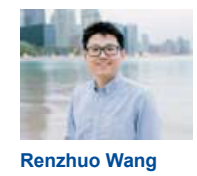

IB1

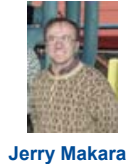

HAB and MC-1

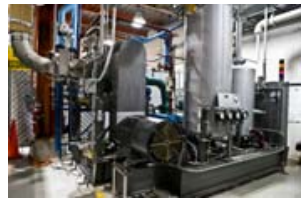

Kinney Vacuum Pump

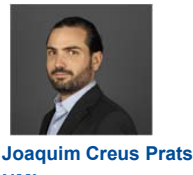

NML

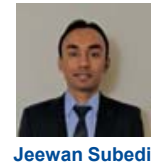

MDB
Industrial Building 1 (IB-1)

Cryogenic System:

- CTI 1500 Cold Box provides up to 300 Liters per hour of liquid helium. The system has 4 Kinney vacuum pumps to enable $2 \mathrm{~K}$ operation

Currently Supports:

- Test Stand 3 - magnet tests including LCLS-II SPQA, Mu2e HTS lead and splice

- Test Stand 4 - being modified to support HILUMI horizontal magnet testing

- LTCF - low temperature calibration of instrumentation including RTDs

- Vertical Test Stands (VTS) - 3 test stands for SRF cavity R\&D and qualification test for LCLS-II and other projects

- Vertical Magnet Test Facility (VMTF) Magnet R\&D and tests
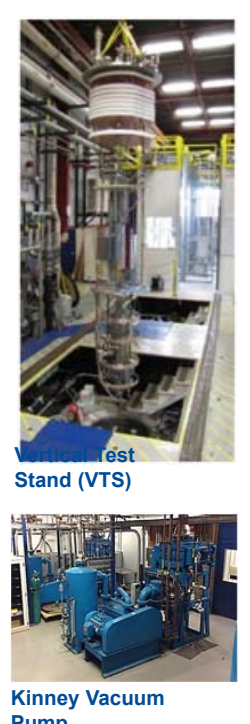
Kinney Vacuum

Meson Cryogenic Test Facility

Cryogenic System:

- Three Tevatron style satellite refrigerators capable of $\sim 1000 \mathrm{~W}$ at $4.5 \mathrm{~K}$. A Kinney vacuum pump enables $2 \mathrm{~K}$ operations to support single cavity loads of 10-20W.

Currently Supports:

- Horizontal Test Stand (HTS) - Integrated cavity tests with fundamental power couplers and tuner, supporting LCLS-II $1.3 \mathrm{GHz}$ and $3.9 \mathrm{GHz}$ testing

- Spoke Test Cryostat (STC) - PIP-II $325 \mathrm{MHz}$ Single Spoke Resonator integrated cavity tests, and being upgraded to test $650 \mathrm{MHz}$ cavities for PIP-II

- Rapid Cycling Magnet Test Stand - International Advanced Accelerator R\&D Fast-Cycling SC Magnets Tests
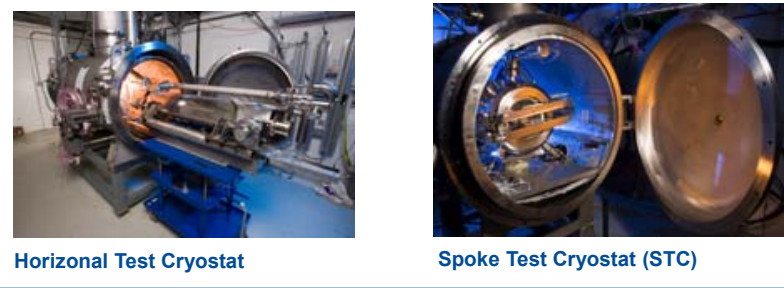

Spoke Test Cryostat (STC)
Heavy Assembly Building (HAB)

Cryogenic System:

The helium cryogenic system consists of two Mycom screw compressors $(60 \mathrm{~g} / \mathrm{s})$ supporting a $600 \mathrm{Watt}$ at $4.5 \mathrm{~K}$ cryogenic plant previously used for the Tevatron Collider Detector Facility (CDF).

\section{Currently Supports:}

Testing of superconducting transport solenoids (14) for the future Mu2e experiment at Muon Campus. The test stand in the CDF pit consists of a cryostat with two removable top heads where solenoids are mounted and then inserted into the cryostat.

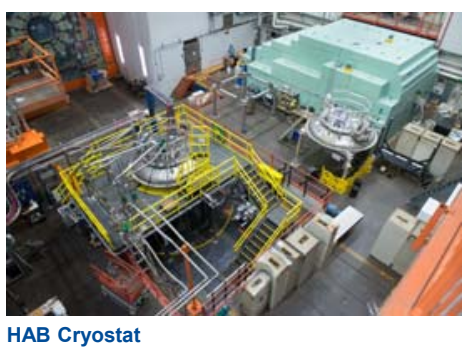

Muon Campus 1 (MC-1)

Cryogenic System:

Consists of four Mycom compressors at $\mathrm{A} 0$ supporting four refurbished Tevatron 600 Watt @4.5 K cryogenic plants at MC-1, Currently Supports:

Provides cooling to the g-2 experiment's superconducting magnet (from Brookhaven) and future Mu2e transport solenoids.

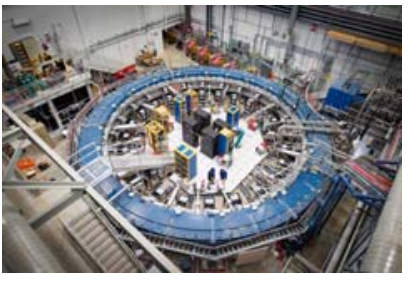

Muon g-2 Experiment

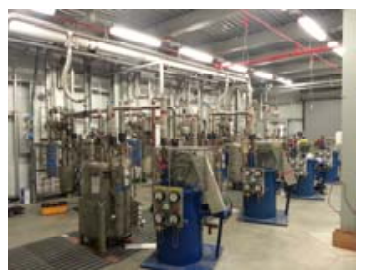

MC-1 Refrigerator Room
This poster has been authored by Fermi Research Alliance, LLC under Contract No. DE-AC02-07CH11359 with the U.S. Department of Energy, Officeof Science, Office of High Energy Physics. 